\title{
GENERATION OF A STATIC POTENTIAL FIELD FOR CAMERA POSITIONING SUPPORT IN A VIRTUAL ENVIRONMENT
}

\author{
Adam Wojciechowski \\ Institute of Computer Science, Technical University of Lodz, Poland; adamwoj@ics.p.lodz.pl
}

\begin{abstract}
This paper introduces an innovative method of a potential field generation. A new construction of the potentials lets the camera be attracted by implicit goals and simultaneously prevents the observer from getting into the object. Proposed method solves the problem of collisions detection without additional implementation. Proposed potential field lets the user come up to a visually selected object quite smoothly and it reduces discrepancy between movement trail and the user's intentions
\end{abstract}

Key words: potential fields, collisions detection, camera navigation, virtual environment

\section{INTRODUCTION}

In this paper potential field construction method is presented. This method is designed to let the user reach implicit goal while navigating quite freely without forcing certain way to the aim. It is also transparent to the user and concurrent with the user's movement intentions. Additionally the user is able to deal with rapidly changing interests in different objects of the scene and is able to reach adequate position and orientation to present the resulting objects in their environment at their usual context and position.

Method proposed in this paper solves the problem of collisions detection and prevents the user from getting into the object what became an additional problem in other authors' solutions.

In section 2 requirements for potential field construction in virtual environment are discussed and the context of necessity of the user's movement support is explained. Section 3 describes related works. In section 
4 theoretical background for constructing universal potential field is presented and moreover, in this part of the paper a new specific way of calculating forces basing on potentials is introduced. In the final section conclusions and ideas for future work are discussed.

\section{POTENTIAL FIELD REQUIREMENTS}

Final requirements concerning potential field construction encompassing possibilities mentioned in the introduction include:

- multiple goal definition,

- existence of attractive forces pushing user towards visually selected goals,

- protection from getting into the object,

- possibility of approaching the object in a smooth and uncomplicated way,

- possibility of changing the goal at any time and position,

- forming one potential field fulfilling all above requirements.

\section{RELATED WORK}

First attitude towards using potential fields in navigation support appeared in robotics. In initially published papers in robotics [2-3] forces, coming out of potential fields, were used generally for obstacle avoidance or determining one predefined and pre-calculated aim (fig.1). It resulted in planning predefined and optimal path from origin to the goal.

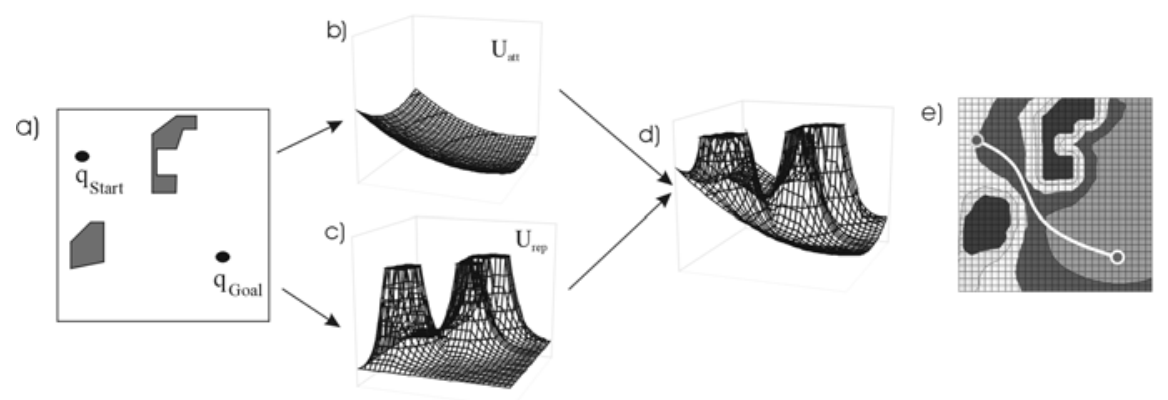

Figure1. Construction of a path (e) basing on objects (a), repulsive potential fields (c), and one predefined goal attracting field (b) $[7,8]$

Quite a new method of a potential stimulated camera navigation in virtual environment was presented in Xiao's [4] paper. As previous works focused 
mainly on fixing a save path to the aim and controlling the point of view after collision with an object, Xiao does not force any certain route but deals with camera obstacle avoidance. Even though Xiao analysed the problem quite thoroughly and proposed many context dependent camera behaviours, he faced some problems regarding collisions with objects and oscillation of a point of view in places where the fields counterbalance.

Continuation of the efforts begun by Xiao [4] is presented in Li [5-6] papers. The author's innovations concern mainly: replacing radial field propagation with wave field propagation determined by shape of the object, different way of collision problem solving, incrementing number of points considered while calculating total influence in order to deteriorate oscillation effect, completing wider context of the user (speed, orientation, history of motion) to select objects which influence should be taken into consideration. Li could not also get rid of collisions calculating problem but he proposed successful method of diminishing field influence while coming up to the object. As a final proposal Li suggested combining the methods based on potential fields and path planning.

\section{POTENTIAL FIELD DEFINITION}

Potential fields are thoroughly discussed in Latombe's work [7]. In this paper just a brief introduction and phenomena characteristics in the context of camera manipulation should be presented.

In described method camera is represented as a point $q$ in configuration space and moves as a particle under the influence of an artificial potential field $U$. The field function $U$ can be defined over the free space as a sum of an attractive potential pulling the particle (camera) to the goal, and a repulsive potential pushing the particle away from the edge of an object. In general, the artificial force could be expressed with the equation (1):

$$
\vec{F}(q)=-\vec{\nabla} \cup(q)
$$

In the newly proposed method, the $U$ function is defined over a discrete space, which reflects complexity of the presented objects. Additionally, function $U$ has just integer values in each node of the mesh (fig.2a). 
a)

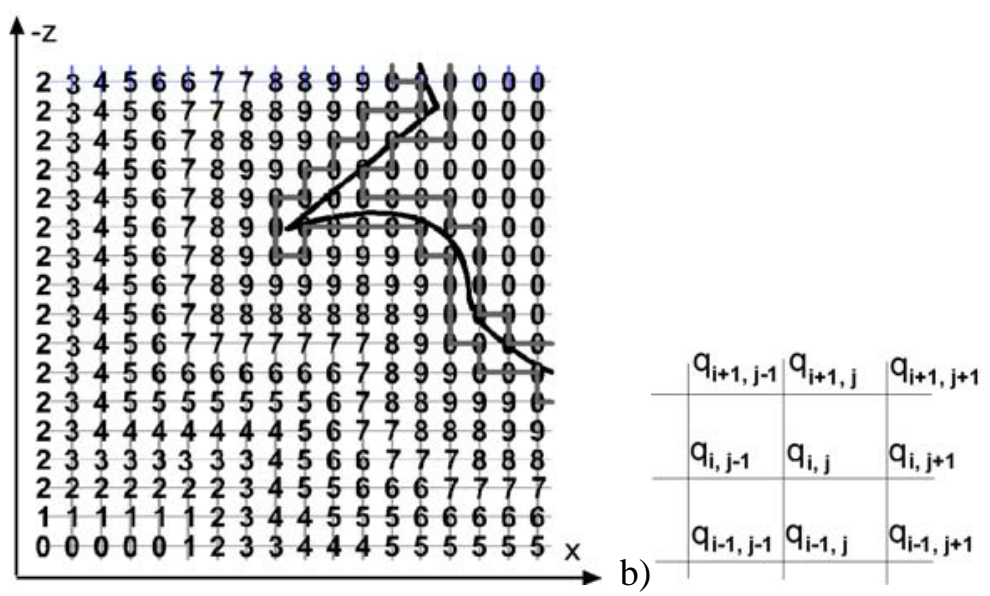

Figure 2. Potential field: a) propagation for a fragment of an exemplary object; b) fragment of a mesh containing node $\mathrm{q}_{\mathrm{i}, \mathrm{j}}$;

In the presence of one goal, the attractive potential $U_{\text {att }}(q)$ can be defined as a conic well (equation 2):

$$
\begin{aligned}
& \left.U_{\text {att }}(q)=\max \left(1 \text {, antie }\left[\left(1-\frac{r}{R}\right) U_{\max }\right]\right) \quad r \in<0,02 R ; R\right) \\
& \left.U_{\text {rep }}(q)=0 \quad r \in<0 ; 0,02 R\right) \\
& U(q)= \begin{cases}1 & r>=R \\
U_{\text {att }}(q) & r \in<0,02 R ; R) \\
U_{\text {rep }}(q) & r \epsilon<0 ; 0,02 R) \\
0 & \text { inside object }\end{cases}
\end{aligned}
$$

where $r$ is the Euclidean distance to the closest edge of the goal and ranges from $R^{*} 0.02$ to $R$ and $R$ is the range of field influence, and $U_{\max }$ is a maximum potential depending on density of a mash and equal for all of the objects in the scene. In a close vicinity of the object, in the distance from 0 to $R^{*} 0.02$, there is just a repulsive potential field $U_{\text {rep }}(q)$ equal to 0 . Inside the object there is also potential equal to 0 but outside the region of influence the potential is equal to 1 . Antie $[x]$ means an integer part of $x$ number. Potential values are positive integers because such attitude fastens calculations and makes presented method more clear.

Summing up total potential $U(q)$ is expressed by equation (4). Equation (4) implicates the fact that attractive and repulsive potentials do not overlap but complement each other. Point $q$ representing a camera is under the influence of just one artificial force coming out of one of the potentials: attractive or repulsive. 
In the presence of multiple objects and goals, the potentials have to be attached in a specific way. They might be joined if the density and orientation of a mesh is identical by taking the maximum value of a node comparing adequate nodes from all of the meshes. In such a case the greatest value of all corresponding nodes is assigned to a target mesh on condition that a node has the value different than zero. Zero means that the taken node lies inside the object and the value cannot be changed.

Such a mounted final mesh can serve as a basis for calculating artificial field $F$. Meshes do not have to be summed up and can be used for forming partial artificial forces $F_{i}$ which influence separately on the moving camera.

Mathematically, a force $F(q)$ got from equation (1) makes use of the gradient what results in calculating quite difficult and time consuming derivatives. In a proposed method field $F$ is estimated in each node $q_{i, j}$ of a mesh, basing on equation (5). Fragment of a mesh with exemplary element $q_{i, j}$ and eight neighbouring elements is presented in a figure $2 \mathrm{~b}$.

$$
\begin{aligned}
& \vec{F}\left(q_{i, j}\right)=\left\langle F_{x}\left(q_{i, j}\right), F_{y}\left(q_{i, j}\right), F_{z}\left(q_{i, j}\right)\right\rangle \\
& F_{x}\left(q_{i, j}\right)=\left(q_{i-1, j+1}-q_{i-1, j-1}+q_{i, j+1}-q_{i, j-1}+q_{i+1, j+1}-q_{i+1, j-1}\right) \\
& F_{y}\left(q_{i, j}\right)=q_{i, j} \\
& F_{z}\left(q_{i, j}\right)=\left(q_{i+1, j-1}-q_{i-1, j-1}+q_{i+1, j}-q_{i-1, j}+q_{i+1, j+1}-q_{i-1, j+1}\right)
\end{aligned}
$$

Node $q_{i, j}$ is situated in row number $i$ and column number $j$ according to left-bottom corner coordinate system (fig.3a,b). Function $F$ in node $q_{i, j}$ of the mesh is a three dimensional function with axis compatible with OpenGL coordinate system. After generating forces for all mesh values $F_{x}\left(q_{i, j}\right)$ and $F_{z}\left(q_{i, j}\right)$ for each node $q_{i, j}$ are normalized. $F_{y}\left(q_{i, j}\right)$ is left unchanged because its value would be utilized for setting camera altitude.

\section{SUMMARY}

The method described above takes advantage of potential fields what is already known attitude. However, the solution met in literature treats potential fields strictly physically with its computing load. Such physical analysis causes certain limitations and problems like these:

- problem of oscillation in points of counterbalancing potential fields,

- local maximums which were placed in accidental places and captured camera attention contradictory to user's intensions,

- physical attitude based separately on either attractive or repulsive forces what caused necessity of analysis of two parallelly existing influences 
- objects were usually treated as obstacles but not as potential goals

- implementing pure potential field did not prevent camera from getting into the object

Contrary to the described above problems, the proposed method forms one universal potential field. It provides at one time both repulsive and attractive forces according to the force necessity. It means that potential force attracts user when he comes up to the object and stops him when he reaches the objects' edge. Additionally potential field is defined in such a way that it does not generate local maximums in accidental places what many authors treated as serious disadvantage of potential field usage. Presented definition of potential field supports also collisions detection what, according to other authors, must be implemented additionally to repulsive and attractive potential fields. Undoubtly, one of big advantages accompanying this method is its mixture of flexibility and low calculation cost. Due to pre-calculation of all forces for each of the node, what was possible in case of a static scene, the camera context needs just 16 values taken from an array which influence camera position. It is much faster than real time calculation of influence generated by objects.

\section{REFERENCES}

[1] S. Beckhaus, "Dynamic Potential Fields for Guided Exploration in Virtual Environments”, doctoral dissertation presented to Otto-von-GueickeUniversitat Magdeburg, September 2002

[2] 0. Khatib, Real-time obstacle avoidance for manipulators and mobile robots, Proc. IEEE Robotics and Automation Conference, 500-505, 1985.

[3] J. Borenstein, Y. Koren, Real-time obstacle avoidance for fast mobile robots, IEEE trans. on Systems, Man, and Cybernetics, 1 g(5),1989.

[4] D. Xiao, R. Hubbold, Navigation Guided by Artificial Force Fields, Proceedings of CHI'98, Los Angeles, USA, 1998

[5] Tsen-Yen Li, Shu-Wei Hsu, An Intelligent 3D User Interface Adapting to User Control Behaviours, Proceedings of the 9th international conference on Intelligent user interface, 13-16 January 2004, Madeira, Funchal, Portugal, pp. 184-190

[6] T.Y. Li, H.C. Chou, Improving Navigation Efficiency with Artificial Force Fields, Proceedings of $200114^{\text {th }}$ IPPR Conference on Computer Vision, Graphics, and Image Processing, Taiwan, 2001

[7] J.C. Latombe, Robot Motion Planning, Kluwer Academic Publ., 1991

[8] R.R. Murphy, Introduction to AI Robotics, The MIT Press, 2000 